\title{
INTERNAL ROTATION, MIXING AND LITHIUM ABUNDANCES
}

\section{BRIAN CHABOYER}

\author{
Steward Observatory \\ University of Arizona \\ Tucson, AZ, USA 85710
}

\begin{abstract}
Lithium is an excellent tracer of mixing in stars as it is destroyed (by nuclear reactions) at a temperature around $\sim 2.5 \times 10^{6} \mathrm{~K}$. The lithium destruction zone is typically located in the radiative region of a star. If the radiative regions are stable, the observed surface value of lithium should remain constant with time. However, comparison of the meteoritic and photospheric $\mathrm{Li}$ abundances in the Sun indicate that the surface abundance of $\mathrm{Li}$ in the Sun has been depleted by more than two orders of magnitude. This is not predicted by solar models and is a long standing problem. Observations of $\mathrm{Li}$ in open clusters indicate that $\mathrm{Li}$ depletion is occurring on the main sequence. Furthermore, there is now compelling observational evidence that a spread of lithium abundances is present in nearly identical stars. This suggests that some transport process is occurring in stellar radiative regions. Helioseismic inversions support this conclusion, for they suggest that standard solar models need to be modified below the base of the convection zone. There are a number of possible theoretical explanations for this transport process. The relation between $\mathrm{Li}$ abundances, rotation rates and the presence of a tidally locked companion along with the observed internal rotation in the Sun indicate that the mixing is most likely induced by rotation. The current status of non-standard (particularly rotational) stellar models which attempt to account for the lithium observations are reviewed.
\end{abstract}




\section{Introduction}

$\mathrm{Li}^{1}$ is a sensitive tracer of mixing in stellar radiative regions as it is easily destroyed at temperatures above $\sim 2.5 \times 10^{6} \mathrm{~K}$. For solar type stars, the Li destruction region is located below the surface convection zone in standard models. As a consequence, standard stellar models predict that Li should not be depleted at the surface of solar type stars. This is a rather robust prediction of stellar evolution theory, which has been known for 40 years (Schwarzschild et al., 1957). However, comparisons between the solar photospheric $\mathrm{Li}$ abundance and the $\mathrm{Li}$ abundance in meteorites show that the Sun has depleted a substantial amount of $\mathrm{Li}$ at its surface (Greenstein \& Richardson, 1951). The solar Li depletion problem has posed a challenge to stellar evolution theory for 40 years, and the solution to this puzzle is still open to debate.

The Sun is unique in that helioseismic observations allow us to probe the interior structure and rotation of the Sun. These observations can put constraints on possible solutions to the solar Li depletion problem, but by themselves solar observations cannot uniquely determine the cause of solar Li depletion. Observations of stellar $\mathrm{Li}$ abundances allow one to study the Li depletion problem as a function of age, metallicity and stellar mass. As such, they provide a powerful test for mechanisms which attempt to explain the solar $\mathrm{Li}$ depletion. The discovery of a large dip in $\mathrm{Li}$ abundances around $6600 \mathrm{~K}$ in the Hyades (Boesgaard \& Tripicco, 1986) was not predicted by theorists, and remains a major challenge to theoretical stellar evolution models. There is increasing evidence that a dispersion in $\mathrm{Li}$ abundances exists among stars with similar ages, metallicities and masses (Soderblom et al. 1993; Boesgaard et al. 1998). Such a dispersion suggests that another stellar property is important in determining the amount of $\mathrm{Li}$ which is depleted in stars. There is mounting observational evidence that rotation plays a key role in determining the amount of Li depletion in a star (Barrado y Navascués \& Stauffer 1997; Jones et al. 1997). In this review, I will discuss the relationship between mixing, rotation and $\mathrm{Li}$ abundances in stars.

\section{Solar Observations}

The present photospheric abundance of $\mathrm{Li}$ in the Sun has been depleted by a factor of $140 \pm 40$ as compared to the meteoritic value of $\log \mathrm{N}(\mathrm{Li})=3.31 \pm 0.04$ (Anders \& Grevesse, 1989) ${ }^{2}$. Solar models which do not allow for transport or mixing in the radiative regions of the Sun predict very little Li depletion (a factor of $2-3$ ). This has been a remarkably robust prediction of standard stellar structure theory which has remained unchanged for 40 years (Schwarzschild et al. 1957; Chaboyer et al. 1995a), despite the fact that the opacities and nuclear reaction rates used in stellar structure codes have changed considerably over this time period. Current solar models imply that the region where $\mathrm{Li}$ is destroyed in the Sun is $\sim 0.05 \mathrm{R}_{\odot}$ below the base of the

\footnotetext{
${ }^{1}$ In this review I will use $\mathrm{Li}$ to represent ${ }^{7} \mathrm{Li}$, the isotope which is produced by big bang nucleosynthesis. ${ }^{7} \mathrm{Li}$ accounts for $\sim 93 \%$ of the total $\mathrm{Li}$ abundance in meteorites. Observers typically measure the total $\mathrm{Li}$ abundance, while theoretical models determine depletion factors for ${ }^{7} \mathrm{Li}$ and ${ }^{6} \mathrm{Li} \mathrm{The}{ }^{6} \mathrm{Li}$ isotope is destroyed at much lower temperatures and ${ }^{7} \mathrm{Li}$. When making the comparison between the observations and theory, it is usually assumed that the ${ }^{6} \mathrm{Li}$ contribution to the stellar $\mathrm{Li}$ content is neglible. However, see the discussion on halo stars (§4) where observations of ${ }^{6} \mathrm{Li}$ may be used to elucidate the mixing mechanism operating in these stars.

${ }^{2}$ Using the standard notation where $\log \mathrm{N}(\mathrm{Li}) \equiv 12+\log [\mathrm{N}(\mathrm{Li}) / \mathrm{N}(\mathrm{H})]$.
} 


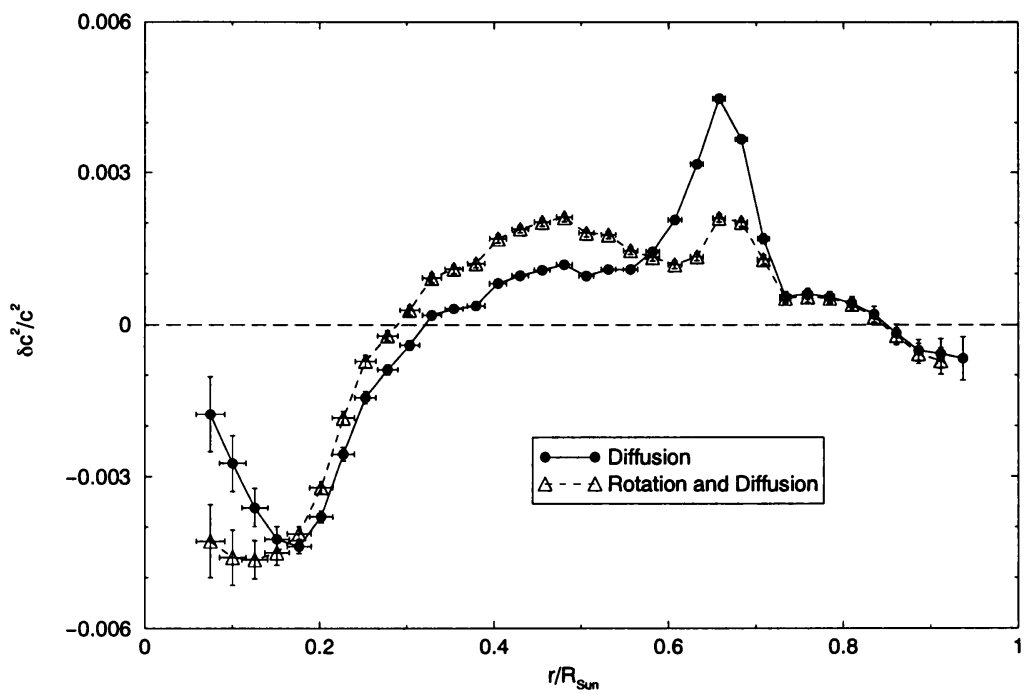

Figure 1. The difference in the square of the sound speed $\left(c^{2}\right)$ between two solar models and the actual Sun, as determined from helioseismology.

solar convection zone. The Li depletion in the Sun suggests that some material from the region where $\mathrm{Li}$ is destroyed has been transported to the convection zone, leading to the observed Li depletion at the surface.

The cause of this transport process is still a matter of debate. Observations of Be can constrain the nature of this transport process, as Be is burned at a higher temperature $\left(3.5 \times 10^{6} \mathrm{~K}\right)$ than $\mathrm{Li}$, and so probes the deeper interior of the Sun. Observations of the photospheric and meteoritic Be abundances suggest that Be has been depleted at the solar surface by a factor of $1.8 \pm 0.5$ (Anders \& Grevesse, 1989). Thus, at the $3 \sigma$ level it appears that the Sun has indeed depleted $\mathrm{Be}$ at its surface. This is supported by the work of King et al. (1997), who found a minimum surface solar Be depletion level of $12 \%$.

Helioseismic observations can be used to probe the interior structure and rotation of the Sun and provide a strong test for stellar evolution theory. The observed frequencies of the solar $p$-modes depend primarily on the sound speed $c$ in the solar interior and so it is relatively straightforward to invert the helioseismic observations to probe the variation of sound speed with depth in the Sun. In order to linearize the problem, this inversion is typically done with respect to a theoretical solar model. The data which are now available from the GONG project (Harvey et al., 1996) and SOHO satellite (Rhodes et al., 1997) allow for a very precise determination of the sound speed in the Sun, down to $r \simeq 0.1 R_{\odot}$. A typical example of a solar sound speed inversion is shown in Figure 1, which shows that current solar models are able to match the observed value of $c^{2}$ to within $0.5 \%$ throughout the solar interior. This is a remarkably achievement for stellar evolution theory, made possible by many advances in the last 5 years in the opacities and equation of state used within stellar evolution codes. Another important advance has been the realization that diffusion (whereby the elements heavier than hydrogen sink to the center of the star) must be included in solar models in order to match the helioseismic observations (Christensen-Dalsgaard et al., 1993; 
Guenther et al., 1996).

Although the match between the helioseismic observations and theoretical models is impressive, the observations are extremely accurate, and the remaining differences between the models and the Sun can be used to continue to improve the physics used in stellar models. Figure 1 indicates that there is a large error in the diffusion model near the base of the solar convection zone $\left(r \sim 0.7 \mathrm{R}_{\odot}\right)$. Detailed studies of this region have suggested that this error is due to the sharp change in the mean molecular weight which occurs at the base of the solar convection zone in models which include diffusion (Gough et al., 1996). The most likely explanation is that some form of slow turbulent mixing is operating below the base of the solar convection zone (Basu, 1997). The model labeled 'rotation and diffusion' in Figure 1 includes slow turbulent mixing generated by rotation (Chaboyer et al., 1995a), and provides a much better match to the observed Sun near the base of the convection zone than the model which only includes diffusion. Helioseismic observations provide strong evidence that slow turbulent mixing occurs below the base of the convection zone in the Sun.

Helioseismology can also probe the interior rotation rate of the Sun. The global modes of oscillation in the Sun can be described by the spherical harmonics, of radial order $n$, degree $\ell$, and horizontal order $m$ (cf. Thompson et al. 1996). If the Sun were spherically symmetric, then the observed oscillations would depend only on $n$ and $\ell$. Rotation breaks the spherical symmetry of the Sun, leading to a splitting of the common $n, \ell$ modes into different $m$ values. The degree of this splitting can be used to infer the solar rotation rate as a function of depth within the Sun. Current data allows for a reliable rotation rate inversions down to $r \sim 0.4 \mathrm{R}_{\odot}$. Rotation rate rate inversions done by a number of authors, using different data sets have all reached essentially the same conclusion. In the the radiative interior there is quasi-rigid rotation, while a latitude-dependent rotation exists in the entire convection zone (Thompson et al., 1996; Corbard et al., 1997; Kosovichev et al., 1997). The transition between these two regimes occurs in a thin layer below the base of the convection zone and is referred to as the solar tachocline. Recent work suggests that the tachocline is very narrow, with a width estimated to be $r=(0.020 \pm 0.005) \mathrm{R}_{\odot}$ (Basu, 1997) or $r=(0.05 \pm 0.03) \mathrm{R}_{\odot}$ (Corbard et al., 1998).

\section{Young Cluster Observations}

Observations of $\mathrm{Li}$ in young cluster stars can be used to empirically determine the amount of stellar Li depletion as a function of age, mass and chemical composition. These observations provide very strong constraints for stellar models. A striking result from early observations of $\mathrm{Li}$ abundances in cluster stars was the existence of a dip in $\mathrm{Li}$ abundances in the $\mathrm{F}$ stars (around $\mathrm{T}_{\text {eff }} \simeq 6600 \mathrm{~K}$ ) in the Hyades cluster (Boesgaard \& Tripicco, 1986). Stars around this temperature range have $\mathrm{Li}$ abundances which are at least 1.0 dex lower than stars which are hotter or cooler than the dip (see Figure 2). This dip was not predicted by theoretical models. Stars on the hot side of the Li dip do not have convective envelopes, and stars on the cool side of the Li dip have small convective envelopes. The Li dip occurs in stars with very small convective envelopes. The Hyades is a somewhat metal-rich cluster $([\mathrm{Fe} / \mathrm{H}]=+0.1$, (Boesgaard \& Friel, 1990)) which is approximately $600 \mathrm{Myr}$ old (Perryman et al., 1998). Observations of $\mathrm{Li}$ abundances in other clusters (the Pleiades in particular) have shown that the $\mathrm{Li}$ dip does not exist for stars on the zero age main sequence (ZAMS) (Soderblom et al., 1993). 


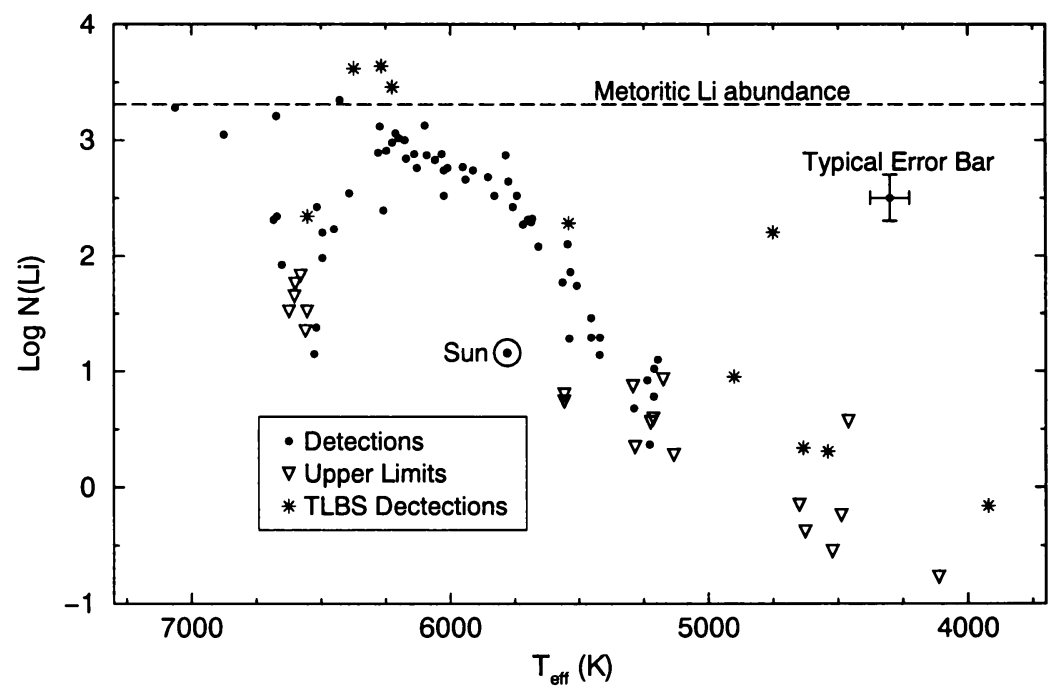

Figure 2. Observations of $\mathrm{Li}$ in the Hyades (Balachandran 1995; Barrado y Navascués \& Stauffer 1997). The photospheric solar $\mathrm{Li}$ abundance is indicated by the solar symbol $\odot$, and the meteoritic $\mathrm{Li}$ abundance is given by the dashed line.

In addition to the Li dip, there are a number of interesting features in Figure 2. In particular, it appears that the tidally locked binary stars (TLBS) have (on average) a higher Li abundance than single stars or binaries which are not tidally locked (Barrado y Navascués \& Stauffer, 1997). This is in good agreement with the theoretical work of Zahn (1994), who postulated that Li depletion is due to rotational mixing and this rotational mixing does not operate in TLBS. The excess $\mathrm{Li}$ abundance observed in TLBS is strong evidence that rotation plays a key role in Li depletion.

The Hyades stars around the solar temperature $\left(T_{\text {eff }}=5780 \mathrm{~K}\right)$ have significantly higher Li abundances in the Sun (Figure 2). This is despite the fact that the Hyades is slightly more metal-rich than the Sun and so theoretical models would predict that the Hyades stars should deplete more Li than the Sun on the pre-main sequence. This suggests that Li depletion in these cooler stars $\left(T_{\text {eff }} \lessgtr 6000 \mathrm{~K}\right.$ ) occurs on the main sequence, a conclusion which is reinforced by the observed Li abundances in the Pleiades (Chaboyer et al., 1995b).

Another interesting feature of Figure 2 is that the highest $\mathrm{Li}$ abundances observed are similar to the meteoritic Li abundances, suggesting that their has been no significant $\mathrm{Li}$ enhancement over the last $4 \mathrm{Gyr}$. It is important to note that the only stars which have $\mathrm{Li}$ abundances similar to the meteoritic abundances are either TLBS, or are on the hot side of the dip (and hence, do not have a surface convection zone). The question then arises, do all single stars with surface convection zones deplete Li? The stars which have the least amount of $\mathrm{Li}$ depletion are those on the cool side of the Li dip, around $\mathrm{T}_{\text {eff }}=6200 \mathrm{~K}$. In an seminal paper, Boesgaard (1991) demonstrated that, even for the stars around $\mathrm{T}_{\text {eff }}=6200 \mathrm{~K}$, there was a clear correlation between their $\mathrm{Li}$ abundance and age. Older stars have lower $\mathrm{Li}$ abundances, implying that all stars with surface convection zones deplete $\mathrm{Li}$ on the main sequence. The work of Boesgaard (1991) utilized observations from a number of different observers, and 


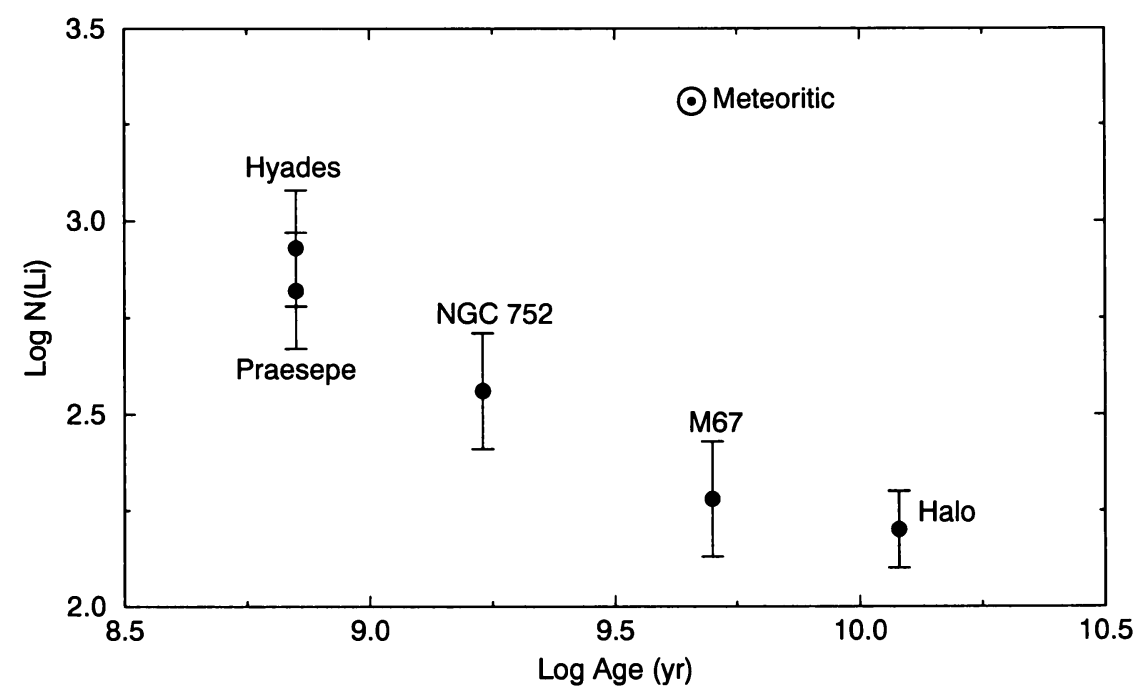

Figure 3. The mean abundance of $\mathrm{Li}$ for stars in the temperature range $6300 \leq \mathrm{T}_{\text {eff }} \leq 6100 \mathrm{~K}$ in four different clusters, as a function of the cluster age (data from Balachandran 1995). The mean abundances for stars in the same temperature range in the halo is taken from the data of Ryan et al. (1996). The meteoritic $\mathrm{Li}$ abundance is also indicated.

included stars with a rather large range in effective temperatures. In order to see if this had any effect on her conclusions, I have repeated the analysis of Boesgaard (1991) using the uniform data set compiled by Balachandran (1995) and only included stars with $6300 \leq \mathrm{T}_{\text {eff }} \leq 6100 \mathrm{~K}$. The results are shown in Figure 3. Even with this restricted data set it is clear that older stars have lower $\mathrm{Li}$ abundances, implying that $\mathrm{Li}$ depletion has occurred on the main sequence for stars around $\mathrm{T}_{\text {eff }}=6200 \mathrm{~K}$. It is interesting to note that halo stars in the same temperature range appear to match the trend found in open cluster stars (see $§ 4)$.

The existence of a dispersion in $\mathrm{Li}$ abundances at a given effective temperature within a cluster has been the subject of a number of observational papers. If such a dispersion exists, it proves that something besides age, mass and chemical composition must control Li depletion in stars. Thorburn et al. (1993) studied in detail the existence of a dispersion among Hyades stars. They found that a such a dispersion does exist for stars with $\mathrm{T}_{\text {eff }}=6100 \mathrm{~K}$, with $\sigma_{\mathrm{N}(\mathrm{Li})} \sim 0.15 \mathrm{dex}$. The existence of a dispersion among the cooler stars was more difficult to prove, though the data did suggest that a small dispersion of $\sigma_{\mathrm{N}(\mathrm{Li})} \sim 0.09$ dex existed among the $\mathrm{G}$ stars $\left(\mathrm{T}_{\text {eff }} \sim 5500 \mathrm{~K}\right)$. In contrast, there is clear evidence for a Li dispersion in this temperature range among the Pleiades (a solar metallicity cluster whose stars are on the ZAMS) stars, while there is little evidence for a dispersion among the hotter Pleiades stars (Soderblom et al., 1993). There was also clear evidence for a correlation between rotation rates and $\mathrm{Li}$ abundances in the Pleiades, with the fastest rotators having the highest Li abundances. A recent paper by Jones et al. (1997) studies in detail the dispersion in $\mathrm{Li}$ abundances (at a given effective temperature) among the Pleiades (age $70 \mathrm{Myr}$ ), M34 (age $250 \mathrm{Myr}$ ) and the Hyades (age $600 \mathrm{Myr}$ ). They found that the dispersion among the lower mass stars $\left(M \sim 0.65\right.$ to $\left.0.95 \mathrm{M}_{\odot}\right)$ is greatest on the 
ZAMS, and decreases with age. The fastest rotating stars have the highest Li abundances. In addition, they found that $\mathrm{Li}$ was depleted on the main sequence, leading Jones et al. to conclude "high rotation preserves lithium during pre-main sequence evolution and that a high angular momentum loss rate accelerates lithium depletion after the star is on the main sequence". This is a key observational fact, which implies that rotation must lead to mixing and Li depletion in low mass main sequence stars.

However, the basic assumption used in the above analysis, that a dispersion in $\mathrm{Li}$ equivalent widths corresponds to a dispersion in $\mathrm{Li}$ abundances has been brought into question by Stuik et al. (1997). These authors suggest that magnetic activity (which leads to the formation of pots and plage on cool stars) may effect the $\mathrm{Li}$ line strength. Thus, the observation that fast rotators have high Li equivalent widths could be due to the fact that fast rotators are more active stars than slow rotators. The work of Stuik et al. (1997) is based upon theoretical models (which have known problems), and is a schematic feasibility analysis, rather than a definitive statement that magnetic activity effects Li line strengths. Nevertheless, it raises a key point which clearly requires more attention in the future.

\section{Halo Star Observations}

Observations of $\mathrm{Li}$ in very old stars has the potential to determine the primordial $\mathrm{Li}$ abundance. $\mathrm{Li}$ is one of only 3 elements (the others being $\mathrm{H}$ and $\mathrm{He}$ ) which are believed to have been produced in significant amounts by big bang nucleosynthesis. Thus, the determination of the primordial Li abundance is a key constraint on theoretical models of big bang nucleosynthesis. In their pioneering work, Spite \& Spite (1982) measured Li abundances in 13 metal-poor (halo) stars, and found that all of the stars had nearly identical $\mathrm{Li}$ abundances. As there was no relation between $\mathrm{Li}$ abundances and metallicity, this suggested that the observed $\mathrm{Li}$ abundance was the primordial $\mathrm{Li}$ abundance. Since then, there have been numerous studies of $\mathrm{Li}$ abundances in halo stars. These studies confirmed that halo stars with $\mathrm{T}_{\text {eff }} \geq 5600 \mathrm{~K}$ have nearly identical Li abundances, independent of their metallicity or temperature (Spite et al., 1984; Rebolo et al., 1988; Hobbs \& Duncan, 1987; Hobbs \& Thorburn, 1991).

However, this assumption was brought into question by the discovery of a number of hot halo stars which have abundances significantly lower than the plateau (Hobbs et al., 1991; Spite et al., 1993; Thorburn \& Beers, 1993; Thorburn, 1992). About 5\% of the halo stars in the plateau effective temperature range have low $\mathrm{Li}$ abundances. These stars demonstrate that at least some hot halo stars do deplete $\mathrm{Li}$, leaving open the possibility that plateau stars may have also depleted $\mathrm{Li}$. The current status of $\mathrm{Li}$ abundances measurements in halo stars is shown in Figure 4.

The existence of a dispersion among the Li plateau stars would strongly suggest that even these stars have depleted $\mathrm{Li}$. The question of weather or not a dispersion exists among the plateau stars is an open one. Deliyannis et al. (1993) performed a dispersion analysis in the $\mathrm{Li}$ equivalent width-colour data and concluded that an intrinsic $\mathrm{Li} / \mathrm{H}$ dispersion of $10 \%$ existed among the plateau stars. Furthermore, a uniform analysis of $\sim 80$ plateau halo stars found a correlation between $\mathrm{Li}$ abundances, effective temperatures and metallicity (Thorburn, 1994). Hotter, and/or more metalrich stars were found to have higher $\mathrm{Li}$ abundances. This work was confirmed by Ryan et al. (1996). Using a sub-set of the above observations, and adopting a different effective temperature scale Molaro et al. (1995) and Bonifacio \& Molaro (1997) found no evidence for a dispersion, or correlation with $[\mathrm{Fe} / \mathrm{H}]$. 


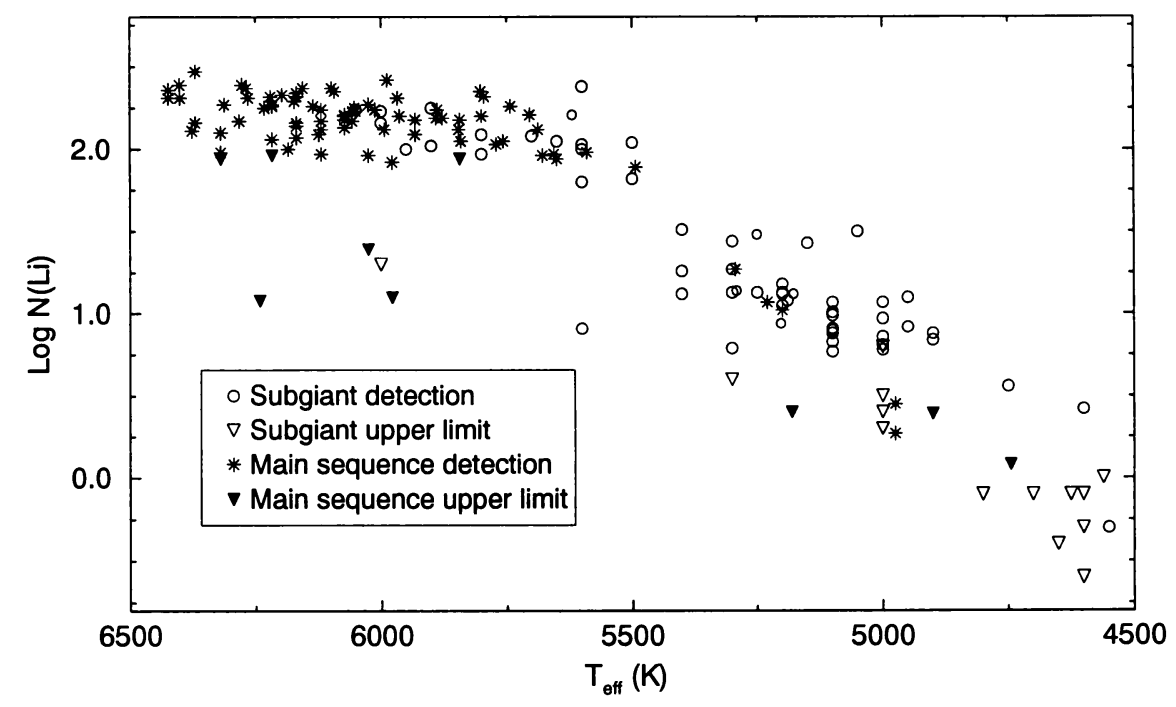

Figure 4. Li abundances in low metallicity $([\mathrm{Fe} / \mathrm{H}]<-1.0)$ field stars (halo stars). Data from Pilawchoski et al. (1993), Thorburn (1994), and Deliyannis et al. (1994).

In order to directly probe for the existence of a $\mathrm{Li}$ dispersion among hot halo stars, Boesgaard et al. (1998) have determined $\mathrm{Li}$ abundances of 7 stars in the globular cluster M92 $([\mathrm{Fe} / \mathrm{H}]=-2.1)$. Stars in a given globular cluster all have the same metallicity, age and reddening. In addition, it is possible to observe a number of stars with virtually the same colour, implying that the stars all have the same effective temperature. Boesgaard et al. (1998) observed 4 stars with virtually identical effective temperatures, of which 1 star has a significantly higher $\mathrm{Li}$ abundance than the others. In all other aspects (including a detailed element by element analysis), the star with the high $\mathrm{Li}$ abundance is virtually identical to the other 3 stars. This observation strongly suggests that a dispersion in $\mathrm{Li}$ abundances exists among hot halo stars and that this dispersion is due to stellar depletion from a higher primordial Li abundance.

Observations of ${ }^{6} \mathrm{Li}$ can also be used to determine if the plateau stars have depleted $\mathrm{Li} .{ }^{6} \mathrm{Li}$ is a destroyed at much lower temperatures than ${ }^{7} \mathrm{Li}$, so that if enough mixing has occurred to deplete ${ }^{7} \mathrm{Li}$, virtually all of the ${ }^{6} \mathrm{Li}$ should be destroyed. An observation of ${ }^{6} \mathrm{Li}$ in a hot halo star would suggest that there has not been any significant depletion of ${ }^{7} \mathrm{Li}$ in these stars, and that the observed ${ }^{7} \mathrm{Li}$ abundance is very close to the primordial abundance ${ }^{3}$. The best evidence for a ${ }^{6} \mathrm{Li}$ detection in a hot halo star has been presented by Smith et al. (1993) and Hobbs \& Thorburn (1997). Both of these groups claim a detection of ${ }^{6} \mathrm{Li}$ in the subgiant $\mathrm{HD} 84937([\mathrm{Fe} / \mathrm{H}]=-2.4$, $\left.\mathrm{T}_{\text {eff }}=6100 \mathrm{~K}\right)$. The careful analysis of Hobbs \& Thorburn (1997) led them to conclude that ${ }^{6} \mathrm{Li} /{ }^{7} \mathrm{Li}=0.08 \pm 0.04$ in HD 84937 . Thus, the detection is significant at

\footnotetext{
${ }^{3}$ Standard big bang nucleosynthesis predicts that all of the $\mathrm{Li}$ production will be in the form of ${ }^{7} \mathrm{Li}$. ${ }^{6} \mathrm{Li}$ can be produced by cosmic ray spallation in the interstellar medium. Deliyannis \& Malaney (1995) have suggested that ${ }^{6} \mathrm{Li}$ may be produced by stellar flares at the surface of the star, implying that an observation of ${ }^{6} \mathrm{Li}$ in the photosphere of a star does not constrain the depletion of ${ }^{7} \mathrm{Li}$ in the stellar interior.
} 
the $2 \sigma$ level. It is premature to conclude that ${ }^{6} \mathrm{Li}$ has definitively been detected in a hot halo star.

Halo stars cooler than the plateau show progressively lower lithium abundances, similar to that seen in open clusters. This is true both for main sequence stars, and subgiant stars (see Figure 4). In both cases, the deepening convective envelopes have brought $\mathrm{Li}$ depleted material to the surface of the star. It is interesting to note that stellar evolution models which incorporate the latest available input physics are unable to correctly reproduce the onset of $\mathrm{Li}$ depletion observed in main sequence or subgiant halo stars (Chaboyer, 1995). This implies that the models are in need of revision, and casts doubt on the ability of the models to correctly predict the amount of ${ }^{6} \mathrm{Li}$ depletion which occurs.

\section{Theoretical Models}

The preceding sections have made it clear that Li depletion has occurred in stars with a variety of masses and ages, for which standard stellar evolution models predict no $\mathrm{Li}$ depletion. A number of mechanisms have been proposed which deplete $\mathrm{Li}$, in order to explain some or all of the $\mathrm{Li}$ observations. These $\mathrm{Li}$ depletion mechanisms include: overshoot, mass loss, mixing induced by gravity waves, diffusion, and mixing induced by rotation.

Overshoot at the base of the convection zone has been proposed by a number of authors (most recently by Ahrens et al. 1992) to explain the depletion of $\mathrm{Li}$ in the Sun. Solar models which include an overshoot of $\sim 0.24$ pressure scale heights at the base of the convection zone are able to reproduce the observed solar Li depletion. However, this $\mathrm{Li}$ depletion occurs on the pre-main sequence, implying very low $\mathrm{Li}$ abundances in young, cool $\left(\mathrm{T}_{\text {eff }} \sim 5800 \mathrm{~K}\right)$ stars. This is not seen in any of the open cluster observations (including the Hyades, Figure 2 and the Pleiades). Open cluster observations rule out fast overshoot at the base of surface convection zones as a viable explanation of the observed solar Li depletion (Chaboyer et al., 1995b).

Large amounts of mass loss $\left(\sim 0.05 \mathrm{M}_{\odot}\right)$ sufficient to expose Li depleted material at the surface of a star has been suggested as a cause of the F star Li dip (Schramm et al., 1990). However, a detailed stellar evolution study by Swenson \& Faulkner (1992) found that mass loss alone could not explain all open cluster Li observations. Furthermore, a detailed study of Be abundances among Li dip stars has detected moderate Be deficiencies among stars with severe (but detected) Li abundances (Stephens et al., 1997). The mass loss hypothesis requires that all of the $\mathrm{Li}$ be depleted before any Be depletion occurs, in contradiction with these observations.

Press (1981) suggested that internal gravity waves generated at the base of the convective envelope of a star may produce weak mixing in the radiative interior. This suggestion was studied in detail by García López \& Spruit (1991), who found that mixing induced by internal gravity waves could explain the Li dip observed in $\mathrm{F}$ stars if the intensity of the gravity waves was increased by a factor of 15 above mixing length estimates. The degree of Be depletion predicted by these observations appears to be incompatible with the observations of Stephens et al. (1997).

The diffusion of $\mathrm{Li}$ out of the surface convection zone was suggested by Michaud (1986) to be the cause of the $\mathrm{Li}$ gap. The models required a small mass loss rate $\left(10^{-15} \mathrm{M}_{\odot} \mathrm{yr}^{-1}\right)$ to explain the lack of $\mathrm{Li}$ depletion on the hot side of the dip. Helioseismology indicates that He diffusion occurs in the Sun $(\S 2)$, indicating that $\mathrm{Li}$ diffusion should also be operating in stars. As the diffusion time scales for $\mathrm{Li}$ and $\mathrm{Be}$ 
are similar, $\mathrm{Be}$ and $\mathrm{Li}$ should be depleted by the same amount if diffusion is the cause of the Li dip. Observations indicate that the degree of Be depletion is much smaller than Li depletion in dip stars (Stephens et al., 1997), ruling out diffusion as the sole cause of the $\mathrm{Li}$ dip. Diffusion is also unable to explain the $\mathrm{Li}$ depletion observed in the cooler stars $\left(\mathrm{T}_{\text {eff }} \lesssim 6000 \mathrm{~K}\right.$ ) like the Sun (Chaboyer et al., 1995b).

It has been known for over 70 years that thermal imbalances in rotating stars give rise to large scale flows (von Zeipel, 1924; Eddington, 1925; Sweet, 1950). Thus, it is not surprising that a number of authors have suggested that the Li observations can be best explained by mixing induced by rotation (e.g. Charbonneau \& Michaud 1988; Vauclair 1988; Pinsonnealt et al. 1990,1992; Charbonnel et al. 1992,1994; Zahn 1992; Chaboyer \& Demarque 1994; Chaboyer et al. 1995a,1995b; Deliyannis \& Pinsonneault 1997). The exact physics underlying rotation induced mixing is still not well understood, leading to a variety of approaches in dealing with the mixing. The Yale rotational models (Pinsonnealt et al. 1990,1992; Chaboyer \& Demarque 1994; Chaboyer et al. 1995a,1995b; Deliyannis \& Pinsonneault 1997) are perhaps the most ambitious, as they self-consistently include the structural effects of rotation along with the coupled transport of angular momentum and material due to a variety of rotation induced mixing mechanisms. These models, along with those of Charbonnel et al. 1994 are able to reproduce a number of features of the observed $\mathrm{Li}$ abundances, including the cool side of the $\mathrm{Li}$ dip and the main sequence depletion of $\mathrm{Li}$ in the Sun and similar temperature cluster stars. Generically, these models also predict a correlation between rotation velocities and $\mathrm{Li}$ abundances (observed in young clusters) and that tidally locked binary stars should not deplete Li (Zahn, 1994) as observed in the Hyades (Barrado y Navascués \& Stauffer, 1997). The rotation models do a good job of matching the Be observations (Stephens et al., 1997) in the Li dip stars (Deliyannis \& Pinsonneault, 1997). These models also predict that a dispersion of Li abundances (due to a dispersion in initial rotation velocities) should exist for stars with equal ages, masses and metallicities, as observed in open clusters.

The small (or non-existent) dispersion among the plateau stars in the halo puts strong constraints on the rotation induced mixing models (Chaboyer \& Demarque, 1994), but does not rule them out. Rotation induced mixing models predict some main sequence Li depletion for all stars with surface convection zones, in good agreement with the observations. By extension, these models also predict significant Li depletion among metal-poor halo stars implying that the primordial abundance is higher than the plateau value (Pinsonnealt et al., 1992; Chaboyer \& Demarque, 1994). The exact amount of Li depletion depends on the details of the models. The Yale models predict a factor of $\sim 10$ depletion, implying a primordial Li abundance of $\log N(\mathrm{Li})=3.1$, a value which is incompatible with standard big bang nucleosynthesis and current estimates for the primordial abundances of helium and deuterium. However, these models do not match all of the observations, and there are considerable uncertainties associated with these models. Thus, the exact amount of Li depletion which has occurred in the plateau stars is still an open problem.

One of the key problems with the Yale rotational models is that angular momentum transport was inefficient, leading to fast rotating cores. This is clearly in contradiction with helioseismic observations of the solar internal rotation (§2). An easy way to correct this problem has recently been identified by Kumar \& Quataert (1997) and Zahn et al. (1997). These authors found that low-frequency gravity waves could be excited by convection in the Sun. These gravity waves can transport angular momentum very efficiently. The estimates for the time scale of mixing by these two groups are very similar to each other, and suggest that rigid body rotation would 
be enforced in the solar radiative region on time scales of $10^{7}$ to $10^{8}$ years. Another difficulty with the Yale models which included the combined effects of rotation and diffusion was that the inclusion of diffusion lead to a differential $\mathrm{Li}$ depletion across the effective temperature of the halo Li plateau (Chaboyer \& Demarque, 1994). The derived $\mathrm{Li}$ abundances did not agree with observations of the most metal-poor stars. This difficulty in reproducing the plateau $\mathrm{Li}$ abundances when diffusion is included can be overcome by the addition of a modest stellar wind $\left(\sim 10^{-12.5} \mathrm{M}_{\odot} \mathrm{yr}^{-1}\right)$ in the models (Vauclair \& Charbonnel, 1995).

\section{Summary}

There is a wealth of data on $\mathrm{Li}$ abundances and rotation velocities in stars with a variety of ages, masses and metallicities. This data clearly indicates that Li depletion occurs on the main sequence for all stars with a surface convection zone. This is in direct contradiction with standard stellar evolution theory. A number of possible mechanisms which lead to extra Li depletion have been put forth. The dispersion in $\mathrm{Li}$ abundances at a given age, metallicity and temperature, the correlation between $\mathrm{Li}$ abundances and rotation velocities in Pleiades, the fact that tidally locked binary stars in the Hyades have an excess Li abundance as compared to single stars, and the detection of moderate $\mathrm{Be}$ deficiencies among $\mathrm{Li}$ dip stars with detectable $\mathrm{Li}$ abundances, all imply that that rotation induced mixing is leading to $\mathrm{Li}$ depletion on the main sequence. Helioseismic observations of the Sun support this hypothesis, for they show that slow form of slow mixing is operating below the base of the solar convection zone (Basu, 1997). Current stellar models which incorporate rotation induced mixing explain many, by not all of the observations. Models which are able to account for all of the data are likely to include diffusion, rotation induced mixing, angular momentum transport by gravity waves and/or magnetic fields and modest stellar winds.

\section{References}

Ahrens, B., Stix, M. \& Thorn, M. 1992, A\&A, 262, 673

Anders, E. \& Grevesse, N. 1989, Geochim. Cosmochim. Acta, 56, 197

Balachandran, S. 1995, ApJ, 446, 203

Barrado y Navascués, D. \& Stauffer, J.R. 1996, A\&A, 310, 879

Basu, S. 1997, MNRAS, 288, 572

Boesgaard, A.M. 1991, ApJ, 370, L95

Boesgaard, A.M., Deliyannis, C.P., Stephens, A. \& King, J.R. 1998, ApJ, 493, 206

Boesgaard, A.M. \& Friel, E.D. 1990, ApJ, 351, 467

Boesgaard, A.M. \& Tripicco, M.J. 1986, ApJ, 302, L49

Bonifacio, P. \& Molaro, P. 1997, MNRAS, 285, 847

Chaboyer, B. 1995, in Stellar Evolution: What Should Be Done?, eds. A. Noels, D. FraipontCaro, M. Gabriel, N. Grevesse \& P. Demarque (Liège: Institut d'Astrophysique), 345 358441,876

Chaboyer, B. \& Demarque, P. 1994, ApJ, 433, 510

Chaboyer, B., Demarque, P. \& Pinsonneault, M.H. 1995, ApJ, 441, 865

Chaboyer, B., Demarque, P. \& Pinsonneault, M.H. 1995, ApJ, 441, 876

Charbonnel, C., Vauclair, S., Maeder, A., Meynet, G. \& Schaller, G. 1994, A\&A, 283, 155

Charbonnel, C., Vauclair, S. \& Zahn, J.-P. 1992, A\&A, 255, 191

Charbonneau, P. \& Michaud, G. 1988, ApJ, 334, 746

Christensen-Dalsgaard, J., Proffitt, C.R. \& Thompson, M.J. 1993, ApJ, 403, L75

Corbard, T., Berthomieu, G., Provost, J. \& Morel, P. 1998, A\&A, 330, 1149 
Corbard, T., Berthomieu, G., Morel, P., Provost, J., Schou, J. \& Tomczyk, S. 1997, A\&A, 324,298

Deliyannis, C.P. \& Malaney, R.A. 1995, ApJ, 453, 810

Deliyannis, C.P., Pinsonneault, M.H. \& Duncan, D.K. 1993, ApJ, 414, 740

Deliyannis, C.P. \& Pinsonneault, M.H. 1997, ApJ, 488, 836

Deliyannis, C.P., Ryan, S.G., Beers, T.C., Thorburn, J.A. 1994, ApJ, 425, L21

Eddington, A.S. 1925, Observatory, 48, 73

García López, R.J. \& Spruit, H.C. 1991, ApJ, 377, 268

Gough, D.O., et al. 1996, Science, 272, 1296

Greenstein, J.L. \& Richardson, R.S. 1951, ApJ, 113, 536

Guenther, D.B., Kim, Y.-C. \& Demarque, P. 1996, ApJ, 463, 382

Harvey, J.W. et al. 1996, Science, 272, 1284

Hobbs, L.M. \& Duncan, D.K. 1987, ApJ, 317, 796

Hobbs, L.M. \& Thorburn, J.A. 1991, ApJ, 375, 116

Hobbs, L.M. \& Thorburn, J.A. 1997, ApJ, 491, 772

Hobbs, L.M., Welty, D.E. \& Thorburn, J.A. 1991, ApJ, 373, L47

Jones, B.F., Fischer, D., Shetrone, M. \& Soderblom, D.R. 1997, AJ, 114, 352

King, J.R., Deliyannis, C.P. \& Moesgaard, A.M. 1997, ApJ, 478, 778

Kumar, P. \& Quataert, E.J. 1997, ApJ, 475, L143

Kosovichev, A.G. et al. 1997, Solar Physics, 170, 43

Michaud, G. 1986, ApJ, 302, 650

Molaro, P., Primas, F., Bonifacio, P. 1994, A\&A, 295, L47

Perryman, M.A.C. et al. 1998, A\&A, 331, 81

Pilachowski, C.A., Sneden, C. \& Booth, J. 1993, ApJ, 407, 699

Pinsonneault, M. H., Deliyannis, C. P. \& Demarque, P. 1992, ApJS, 78, 179

Pinsonnealt, M. H., Kawaler, S. D. \& Demarque, P. 1990, ApJS, 74, 501

Press, W.H. 1981, ApJ, 245, 286

Rebolo, R., Moloaro, P. \& Beckman, J.E. 1988, A\&A, 192, 192

Rhodes, Jr. E.J., Kosovichev, A.G., Scherrer, P.H., Schou, J. \& Reiter, J. 1997, Solar Physics, 175,208

Ryan, S.G., Beers, T.C., Deliyannis, C.P. \& Thorburn, J.A. 1996, ApJ, 458, 543

Schramm, D.N., Steigman, G. \& Dearborn, D.S.P. 1990, ApJ, 359, L55

Schwarzschild, M., Howard, R. \& Härm, R. 1957, ApJ, 125, 233

Soderblom, D.R., Jones, B.F., Balachandran, S., Stauffer, J.R., Duncan, D.K., Fedele, S.B. \& Hudon, J.D. 1993, AJ, 106, 1059

Smith, V.V., Lambert, D.L. \& Nissen, P. 1993, ApJ, 408, 262

Spite, F. \& Spite, M. 1982, A\&A, 115, 357

Spite, M., Molaro, P., Fran cois, P. \& Spite, F. 1993, A\&A, 271, L1

Spite, M., Maillard, J.P. \& Spite, F. 1984, A\&A, 141, 56

Stephans, A., Boesgaard, A.M., King, J.R. \& Deliyannis, C.P. 1997, ApJ, 491, 339

Stuik, R., Bruls, J.H.M.J. \& Rutten, R.J. 1997, A\&A, 322, 911

Sweet, P.A. 1950, MNRAS, 110, 548

Swenson, F.J. \& Faulkner, J. 1992, ApJ, 395, 654

Thompson, M.J., et al. 1996, Science, 272, 1300

Thorburn, J.A. 1992, ApJ, 399, L83

Thorburn, J.A. 1994, ApJ, 421, 318

Thorburn, J.A. \& Beers, T.C. 1993, ApJ, 404, L13

Thorburn, J.A., Hobbs, L.M., Deliyannis, C.P. \& Pinsonneault, M.H. 1993, ApJ, 415, 150

Vauclair, S. 1988, ApJ, 335, 971

Vauclair, S. \& Charbonnel, C. 1995, A\&A, 295, 715

von Zeipel, H. 1924, MNRAS, 84, 665

Zahn, J.-P. 1991, A\&A, 265, 115

Zahn, J.-P. 1994, A\&A, 288, 829

Zahn, J.-P., Talon, S. \& Matias, J. 1997, A\&A, 322, 320 